\title{
Mobile Augmented Reality for Campus Visualization Using Markerless Tracking in an Indonesian Private University
}

\author{
https://doi.org/10.3991/ijim.v15i11.20697 \\ Budi Arifitama $\left.{ }^{\varpi}\right)$, Ghali Hanan, Muhammad Halim Rofiqi \\ Universitas Trilogi, Jakarta, Indonesia \\ budiarifetrilogi.ac.id
}

\begin{abstract}
Marker in augmented reality plays a major part to initiate a virtual object in an augmented environment. Choosing a correct and reliable marker would increase the chance of creating a more stable augmented object especially for visualizing a building structure. Unfortunately, most research on visualizing building structure uses a marker-based tracking approach where users must always bring a pre-printed paper as a tracking media. This creates a problem for every time users demonstrate a specific augmented structure object, they still need to bring a printed marker to show the augmented object. The purpose of this research is to investigate the applicability of markerless-based tracking as a solution whether it can substitute the marker-based tracking on augmented reality problems. Simultaneous Localization and Mapping (SLAM) is used as a markerless tracking method where it tracked and mapped the surface environment using feature extraction, then set an anchor at the specified location where the augmented object visualization appears. The results of the research found that from a total of 30 object detection tests, 21 objects are successfully detected and 9 undetected objects, this shows that markerless-based tracking is applicable and can substitute marker-based tracking for a structural campus visualization.
\end{abstract}

Keywords-Augmented Reality, Structure Visualization, Markerless Tracking

\section{Introduction}

Visualization of a campus structure is important for introducing the building location to new visitors who are still unfamiliar with the campus area. Several media have been used to introduce campus buildings in the form of pictures and maps. However, to provide an imaginable building structure, images are unable to accommodate a real-time visualization to such extent that an innovation is needed to provide a more realistic experience, that is by utilizing augmented reality technology. Augmented reality is an emerging technology that is currently being developed and widely applied in various fields. This technology makes it possible to display a virtual object in a real-time environment as users can interact with the objects to gain knowledge. Marker-based tracking is usually applied to visualize an augmented object, although the dependency of a pre-printed marker that is required to be present and trigger the augmented object, 
makes it inefficient. Therefore, this research was conducted to investigate whether a markerless-based tracking is applicable to replace marker-based tracking as an effort to improving user knowledge of the building structure of a university. Augmented reality has been widely applied as a supporting medium used for a company and organization promotion [1], [2]. In fact, there is an increase in user numbers where augmented reality is used to learn product information prior to purchase [3], [4]. In this research, Simultaneous Localization and Mapping (SLAM) was utilized as a markerless-based tracking solution based on the problem stated, where ground tracking was performed to eliminate the dependence of a marker. This method extracted the surrounding ground environment and used it as a location for the marker to augment a $3 \mathrm{~d}$ object. Testing was performed on markerless-based tracking to validate the performance of the marker by measuring the distance and angle from the marker. This paper is organized as follows. Section 2 describes the related work from previous related research. Section 3 explains about research methodology. Section 4 discusses the results obtained. Last but not least, section 5 describes the conclusion of the research.

\section{Related Works}

Previous research on augmented reality has been developed using both marker-based tracking and markerless-based tracking methods as promotional and informative media to generate interaction with users on learning about a product. Marker-based tracking is commonly used during several sessions, where flyers are used as a marker within interaction sessions and users would aim at the marker to trigger the augmented object for further interaction. Unfortunately, the use of marker-based tracking shows that their dependence on markers makes it inefficient as they always need to prepare markers in advance. In the early years of augmented reality development, marker-based tracking has been used as an approach for object augmentation by using a paper as a location of the marker [5], [6]. One of the research using marker-based tracking explains the application of augmented reality as an effort to introduce traditional cloth from Timor Leste, where the marker in this study is a traditional cloth image [7]. Another research performed using marker-based tracking is in a form of integration of augmented reality technology for education as a part of learning in-class sessions, where a marker was used for tracking augmented objects to help students solve the given problem [8]. However, the dependence on markers that must be printed and carried everywhere makes marker-based less efficient. Therefore, we need a solution to overcome this, namely by applying markerless-based tracking. There are fundamental differences between the two methods. Marker-based tracking requires a medium to be able to show the location of an augmented object, whereas tracking using markerless-based tracking is done without the need for physical media, but tracking is done by extracting points at the surrounding environment to be used as a marker, and users only need to scan at the location. Studies on the use of markerless-based tracking have been carried out based on different problems. One such research aimed to demonstrate the position of the deities in the Balinese Hinduism religion, which used gyroscopes to determine the exact coordinate where each deity appeared [9]. It was confirmed that the use of 
markerless-based tracking are helpful for learners to gain knowledge of the deity position. Augmented reality is very well applied to increase knowledge of product information. The use of markerless tracking provides convenience in the process of transferring knowledge to users, where the dependence on markers can be minimized. Research on retail with the use of markerless tracking [10] was proven to enhancing customer experience with retail products and also assisting in a decision-making process on choosing apparel. Markerless tracking is appropriate for problems that do not require a marker as a medium. In this study, Ground Tracking using Simultaneous Localization and Mapping (SLAM) was used as a method for markerless ground tracking. SLAM is a method that can identify ground areas by building simultaneously a static map of the surrounding environments for the marker location creation [11]. The use of SLAM on markerless tracking eliminates the boundaries of allocating tags on the located area [12]. Markerless tracking has a powerful impact on the augmented reality user experience. It can replace part of the real environment as a marker to pose a virtual object [13]. This tracking system could be achieved using various sensor technologies such as GPS, hybrid vision, gyroscopes, and infrared tags [14].

Research on SLAM is seen applied in museums and galleries [15], where the augmented objects would appear by visiting parts of the exhibition located in the room, and users at the designated location would scan the surrounding environment using SLAM for further augmented interaction. As supporting material, research found the response of users experiencing the application of markerless tracking in museums [16]. It produces information that as many as 26 respondents stated that markerless tracking has a positive impact and provides a good contribution as part of the transfer of information on a product.

\section{$3 \quad$ Research Methodology}

The following section describes the mobile augmented reality scheme, followed by an explanation of the SLAM pipeline implemented, and also how the testing was performed.

\subsection{Mobile augmented reality scheme}

The software development of an augmented reality must go through two stages. The first stage is the back-end, where the software development is performed. The second stage is called the front-end, where the users utilize the mobile augmented reality software developed as seen in Fig. 1. 


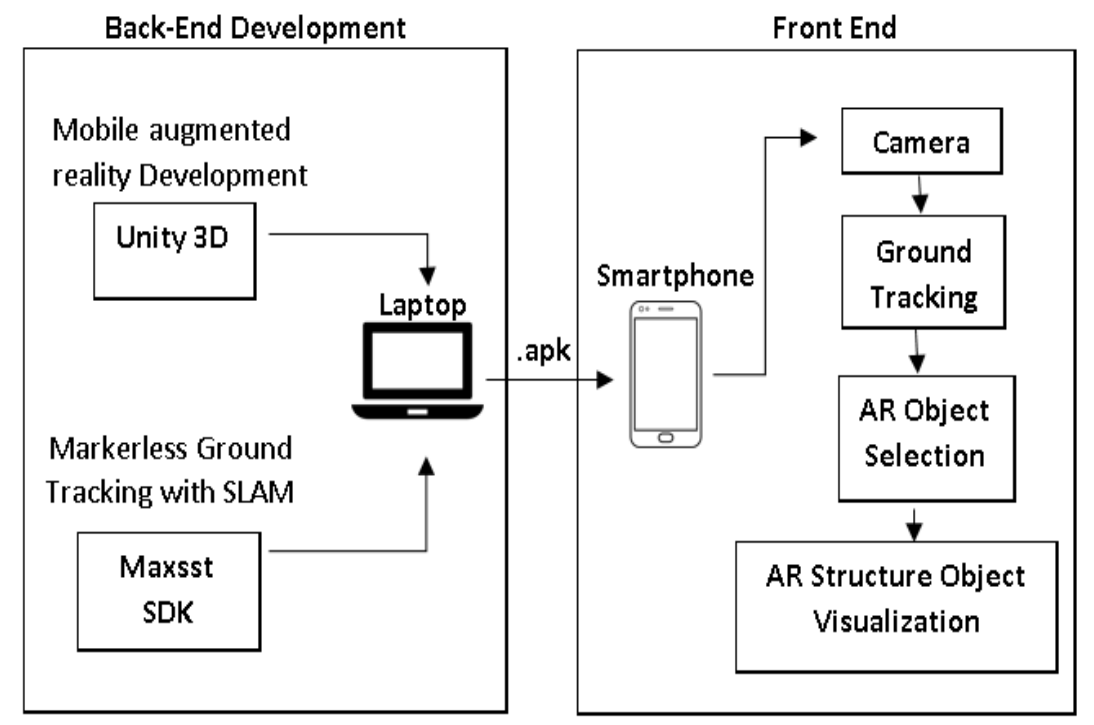

Fig. 1. Mobile augmented reality scheme

Based on the scheme in Fig. 1, it can be seen that at the backend stage, a software development process was carried out using Unity. The implementation of markerlessbased tracking using Simultaneous Localization and Mapping (SLAM) was implemented with Maxxst SDK. The Front-end stage was the stage of the user side of mobile augmented reality. The users used a smartphone embedded with an augmented reality application, then scanned the area that would be marked as the location of the augmented object's appearance. The marker was scanned using SLAM as a method of marker tracking as the location for an augmentable object. After tracking was performed, the augmented object could be visualized on the flat plane of the location desired by the user.

\subsection{SLAM method pipeline scheme}

Simultaneous Localization and Mapping (SLAM) was used as an approach in this study. SLAM can track the marker without having to make a marker first, making it suitable to be applied to replace marker-based tracking on the media used as promotion. 


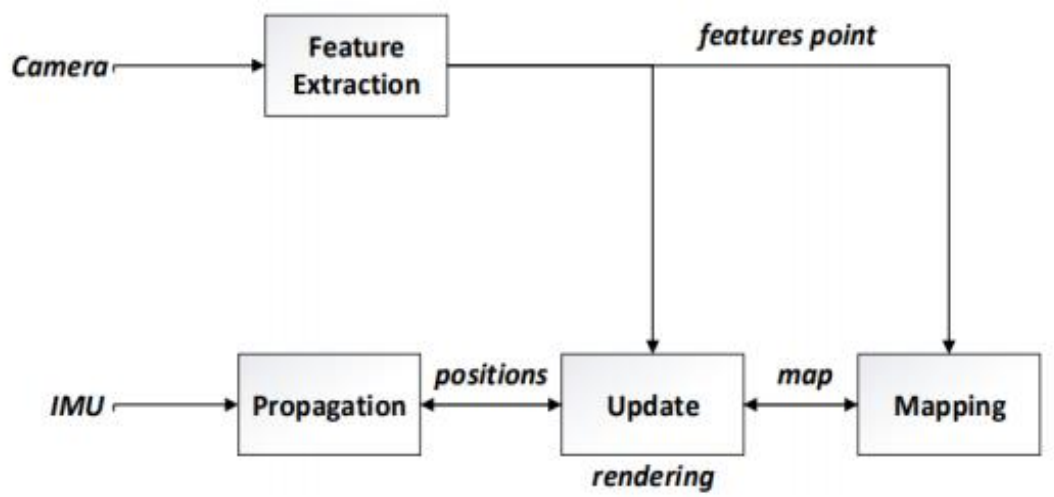

Fig. 2. SLAM pipeline scheme

Fig. 2 is the flow of the stages contained in the process of utilizing SLAM method which has four stages: feature extraction, propagation, mapping, and updates. The following is the SLAM pipeline.

a) Feature extraction: In this stage, the image captured by the camera would be tracked by the system and was given several feature points based on unique elements in every image such as high-contrast spots, curves, or edges. Feature points were points that had been tracked by the system on images captured by the camera to be made as an anchor and had a function as a special marker. Furthermore, the extracted feature points could then be used when mapping to expand the map as agents explore the environment.

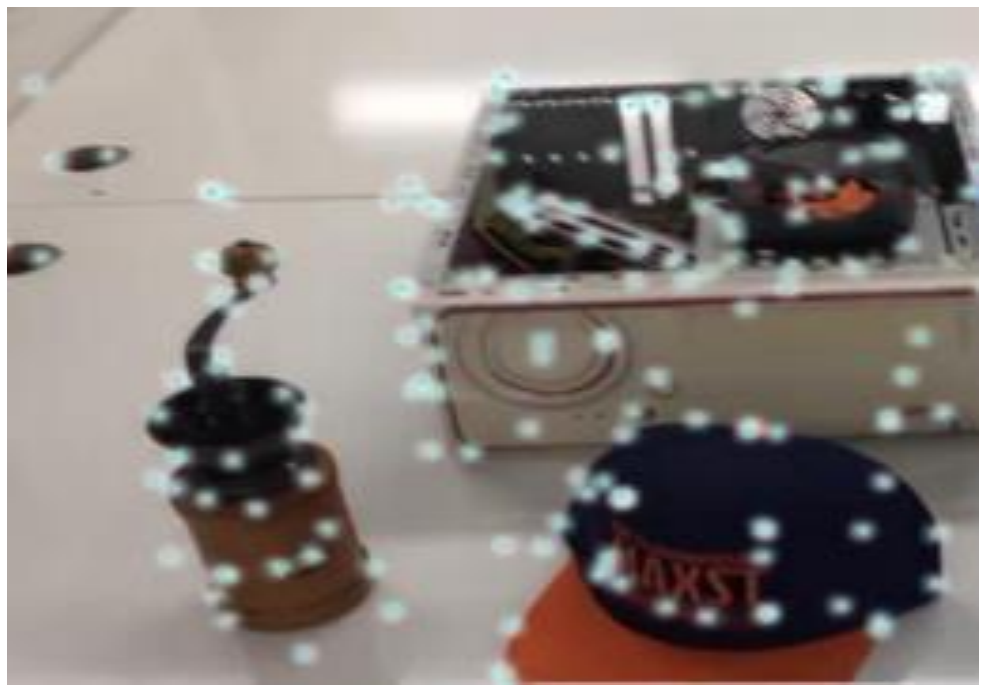

Fig. 3. Feature points extraction 
Fig 3, is a successful result of features point display tracked by the system in the scanned environment which then will perform the triangulation in the propagation stage.

b) Propagation: The main task at the propagation stage was to generate new positions and integrate IMU data points in the form of device position data. From the device side, tracking by using IMU sensor data was useful for estimating poses shown in Fig. 4.

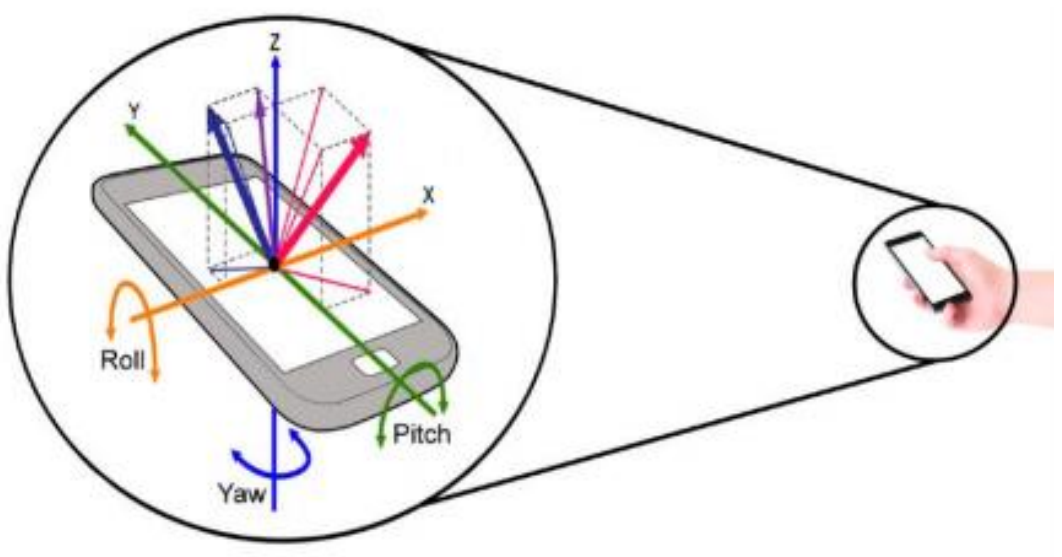

Fig. 4. IMU sensor measurement

Fig. 4 is the Inertial Measurement Unit or IMU sensor used to measure threedimensional motion objects. IMU has a Six Degree of Freedom (6DOF), consisting of three gyroscope axes (pitch, roll, and yaw axes) and three accelerometer axes (x, $\mathrm{y}$, and $\mathrm{z}$ axes) which have their respective uses including gyroscopes to detect tilt angles and measure angular changes of the tilt axis and accelerometer to measure and identify the accuracy of the direction of a device.

c) Mapping: In the mapping stage, the image captured in the frame as the reference image was only processed once when looking for feature points to be used as an anchor. If the feature points detected in the image were few, chances were the 3D model that had appeared unstable was standing on the surface. And then, the feature extraction process would be completed again in the same way and match the new feature point set with the previous one. If most of the feature points were found again in the same position on the frame, the system would store a collection of relative positions of all feature points obtained and triangulate to unite them into something that resembled the shape of 'constellations' so that the feature points did not change significantly when the camera was pointing at it from a different angle. 


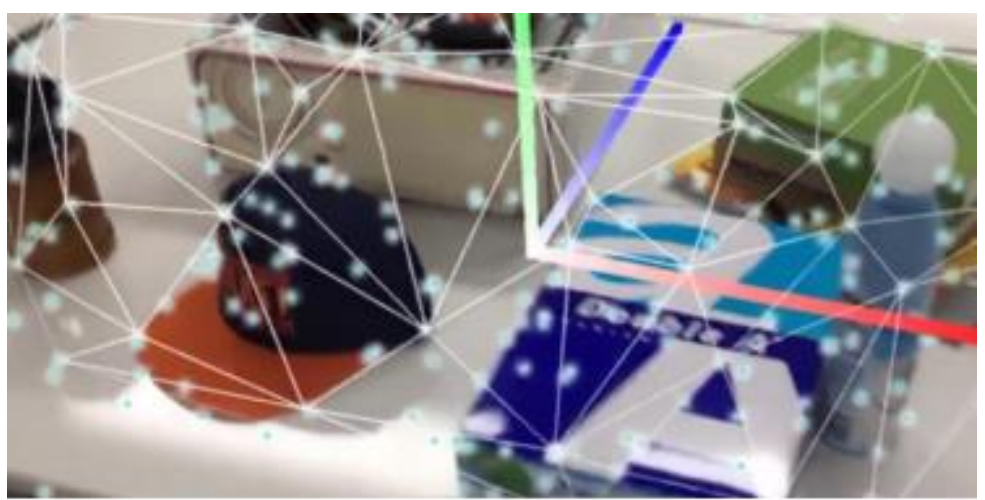

Fig. 5. is a feature point display after performing the triangulation, where the goal is to connect a united line to form a triangle that is used as a container for the augmented model.

d) Updating: Feature points that had been detected in the feature extraction process would be updated by comparing feature points on the map. An update was made to improve the drift introduced by propagation and would update the map with new feature points detected. Next, entry registration used the tracking mechanism to align the virtual content with the real world. The process started with aligning virtual content with the real world, looking for the best camera angle position, the user entering the 3D model and changes the camera's view (to see the 3D model more clearly), and then the system would track the position of the pose based on the movement of the smartphone.

\subsection{D Building model asset collection}

The 3D model structure collection used in this research is based on previous research performed on mobile augmented reality using marker-based tracking obtained from the Departement of Informatics, Universitas Trilogi which has been used as an informative medium since 2015. Fig. 3 shows data about parts of the building that serve as 3D models for augmented reality which consist of the rectorate building, computer lab, and classroom.

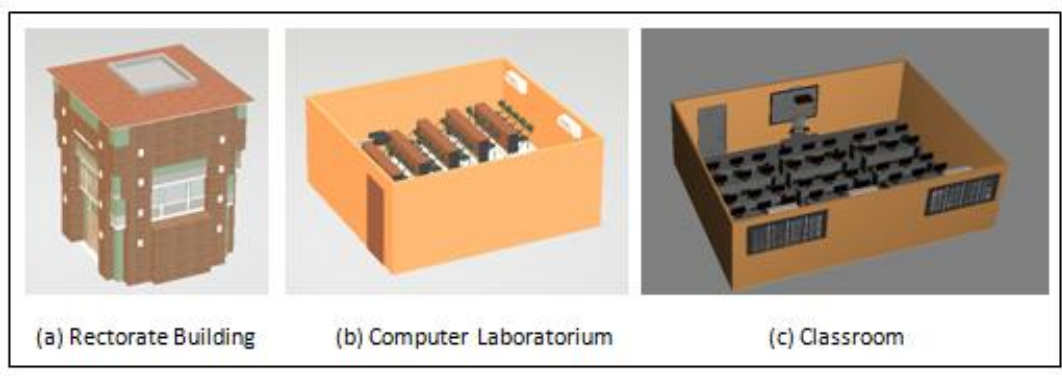

Fig. 6. 3D model structure data collection 


\subsection{Detection testing scenario}

Detection testing experiments were done by using a table as the basis for the appearance of augmented reality objects and as a basis for tracking location from the application of markerless tracking. The parameters used for testing were based on distance and angle measurements as shown in Table 1.

Table 1. Detection testing parameter

\begin{tabular}{|c|c|c|c|}
\hline No & Name & Distance $(\mathrm{cm})$ & Angle (degree) \\
\hline \multirow{10}{*}{1} & \multirow{10}{*}{ Rectorate Building Structure } & 10 & 45 \\
\hline & & 10 & 90 \\
\hline & & 20 & 45 \\
\hline & & 20 & 90 \\
\hline & & 30 & 45 \\
\hline & & 30 & 90 \\
\hline & & 40 & 45 \\
\hline & & 40 & 90 \\
\hline & & 50 & 45 \\
\hline & & 50 & 90 \\
\hline \multirow{10}{*}{2} & \multirow{10}{*}{ Classsroom Building Structure } & 10 & 45 \\
\hline & & 10 & 90 \\
\hline & & 20 & 45 \\
\hline & & 20 & 90 \\
\hline & & 30 & 45 \\
\hline & & 30 & 90 \\
\hline & & 40 & 45 \\
\hline & & 40 & 90 \\
\hline & & 50 & 45 \\
\hline & & 50 & 90 \\
\hline \multirow{10}{*}{3} & \multirow{10}{*}{ Computer Lab Building Structure } & 10 & 45 \\
\hline & & 10 & 90 \\
\hline & & 20 & 45 \\
\hline & & 20 & 90 \\
\hline & & 30 & 45 \\
\hline & & 30 & 90 \\
\hline & & 40 & 45 \\
\hline & & 40 & 90 \\
\hline & & 50 & 45 \\
\hline & & 50 & 90 \\
\hline
\end{tabular}

Based on the parameters in Table 1, the testing process was carried out to obtain data on the response time and the success of object detection from each distance and angle. The distance from the marker tested comprised the shortest standing position of the camera at $10 \mathrm{~cm}$ and the farthest at $50 \mathrm{~cm}$. The two angles tested were 45 degrees and 90 degrees from the position of the marker on the camera. 


\section{$4 \quad$ Results and Discussion}

\subsection{Markerless-based augmented reality implementation}

In this mobile augmented reality application, users detected the object by pointing the camera towards the floor, and then the Simultaneous Localization and Mapping (SLAM) would generate feature points that would determine the location of augmented reality, where users could choose which object to see and interact.

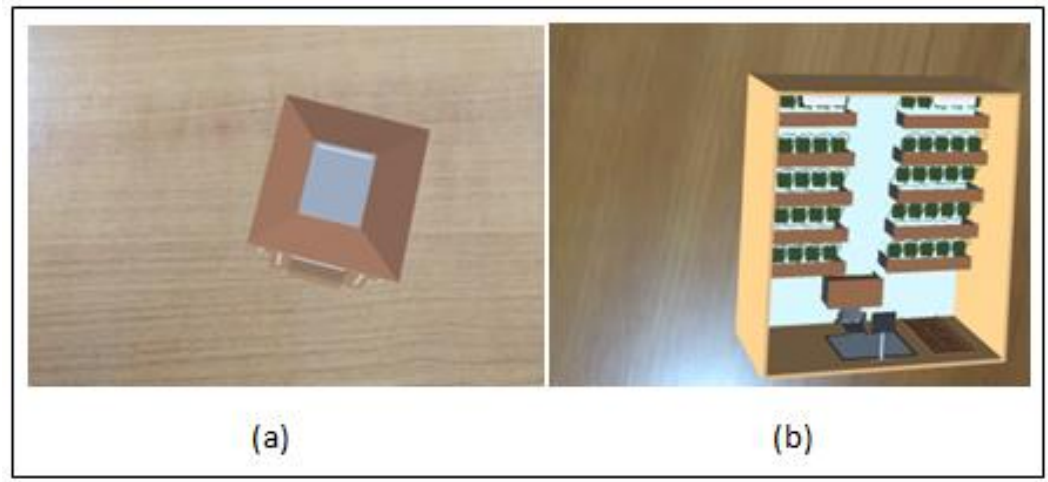

Fig. 7. Augmentable Object Using SLAM Method

Figure 4 (a) and (b) show the structure of the rectorate building and the classroom. In this picture, users could interact by recognizing the visual form of the building and find information about every facility available at the university. Markerless tracking was used to eliminate dependency on the usage of physical markers to access information.

Software used to develop the augmented reality environment are:

- Unity 3D: This is a basic platform for augmented reality development

- Maxst SDK: This is a software development kit for the development of the Markerless Tracking library

- Sketchup: This is used to create a 3D asset design

Hardware used to develop and test the augmented reality object, implement and testing the project are laptop and Smartphones with the following specification:

- Android 9.0 Pie

- 4 GB RAM

- Octa-Core $1.80 \mathrm{GHz}$ Processor 


\subsection{Augmented object detection testing}

Object detection testing is performed by measuring the distance, degree, and response time of the $3 \mathrm{~d}$ object on a markerless tracking that can be seen in Table 2 .

Table 2. Detection testing results

\begin{tabular}{|c|c|c|c|c|c|}
\hline No & Name & Distance & Angle & Time Response & Detection \\
\hline \multirow{10}{*}{1} & \multirow{10}{*}{ Rectorate Building Structure } & 10 & 45 & - & Undetected \\
\hline & & 10 & 90 & - & Undetected \\
\hline & & 20 & 45 & - & Undetected \\
\hline & & 20 & 90 & 1.52 & Detected \\
\hline & & 30 & 45 & 1.23 & Detected \\
\hline & & 30 & 90 & 1.34 & Detected \\
\hline & & 40 & 45 & 1.02 & Detected \\
\hline & & 40 & 90 & 1.02 & Detected \\
\hline & & 50 & 45 & 1.02 & Detected \\
\hline & & 50 & 90 & 1 & Detected \\
\hline \multirow{10}{*}{2} & \multirow{10}{*}{ Classroom Building Structure } & 10 & 45 & - & Undetected \\
\hline & & 10 & 90 & - & Undetected \\
\hline & & 20 & 45 & - & Undetected \\
\hline & & 20 & 90 & 1.72 & Detected \\
\hline & & 30 & 45 & 1.62 & Detected \\
\hline & & 30 & 90 & 1.42 & Detected \\
\hline & & 40 & 45 & 1.02 & Detected \\
\hline & & 40 & 90 & 1.02 & Detected \\
\hline & & 50 & 45 & 1.02 & Detected \\
\hline & & 50 & 90 & 1.2 & Detected \\
\hline \multirow{10}{*}{3} & \multirow{10}{*}{$\begin{array}{l}\text { Computer Lab Building } \\
\text { Structure }\end{array}$} & 10 & 45 & - & Undetected \\
\hline & & 10 & 90 & - & Undetected \\
\hline & & 20 & 45 & - & Undetected \\
\hline & & 20 & 90 & 1.05 & Detected \\
\hline & & 30 & 45 & 1.05 & Detected \\
\hline & & 30 & 90 & 1.04 & Detected \\
\hline & & 40 & 45 & 1.04 & Detected \\
\hline & & 40 & 90 & 1.02 & Detected \\
\hline & & 50 & 45 & 1.03 & Detected \\
\hline & & 50 & 90 & 1.02 & Detected \\
\hline
\end{tabular}

The testing results showed in Table 2 indicate that from a total of 30 experiments conducted, as many as 21 augmented reality objects could be detected by using markerless tracking, and a total of 9 experiments were not detected. A comprehensive calculation of the test using the equation is as follows.

$$
\text { Total Accuracy }=\frac{\text { The number of detected experiments }}{\text { The number of experiments }} \times 100
$$

So, the overall total detection accuracy is: $\frac{21}{30} \times 100=70 \%$. 
A comprehensive calculation to find the average distance accuracy test based on Table 2 is using the equation as follows:

$$
\text { Average Distance Accuracy }=\frac{\text { Amount of Detected Object }}{\text { Amount of Equal Distance }} \times 100
$$

Average distance accuracy testing is performed by measuring the amount of equal distance, and the amount of detected object on a markerless tracking that can be seen on Table 3.

Table 3. Average distance accuracy toward angle

\begin{tabular}{|c|c|c|c|c|}
\hline No & Distance Detection (Cm) & $\begin{array}{c}\text { Amount of Equal Dis- } \\
\text { tances }\end{array}$ & $\begin{array}{c}\text { Amount of detected } \\
\text { object }\end{array}$ & $\begin{array}{c}\text { Average Accuracy } \\
\text { Percentage (\%) }\end{array}$ \\
\hline 1 & \pm 10 & 6 & 0 & $0 \%$ \\
\hline 2 & \pm 20 & 6 & 3 & $50 \%$ \\
\hline 3 & \pm 30 & 6 & 6 & $100 \%$ \\
\hline 4 & \pm 40 & 6 & 6 & $100 \%$ \\
\hline 5 & \pm 50 & 6 & 6 & $100 \%$ \\
\hline
\end{tabular}

A comprehensive calculation to find the average angle accuracy test based on Table 2 is using the equation as follows:

$$
\text { Average Angle Accuracy }=\frac{\text { Amount of Detected Object }}{\text { Amount of Equal Distance }} \times 100
$$

Average angle testing is performed by measuring the amount of detected object with, and the amount of equal distance on a markerless tracking that can be seen in Table 4 .

Table 4. Average angle accuracy toward distance

\begin{tabular}{|c|c|c|c|c|}
\hline No & Angle & Amount of Equal angle & Amount of detected object & $\begin{array}{c}\text { Average Accuracy Percent- } \\
\text { age (\%) }\end{array}$ \\
\hline 1 & 45 & 15 & 9 & $60 \%$ \\
\hline 2 & 90 & 15 & 12 & $80 \%$ \\
\hline
\end{tabular}

A comprehensive calculation performed in Table 4 to measure the average distance accuracy toward angle using the equation is as follows.

$$
\text { Average Angle Accuracy }=\frac{\text { Amount of Detected Object }}{\text { Amount of Equal Angle }} \times 100
$$

Based on the detection testing the results show that the average object detection is $70 \%$. The worst detection distance-based on the test is $10 \mathrm{~cm}$ and the best distance detection is over $40 \mathrm{~cm}$, and the worst distance degree is 45 degrees and the best is 90 degrees. 


\section{Conclusion}

In compliance with the results of the research, it can be concluded that markerless tracking using Simultaneous Localization and Mapping (SLAM) can detect augmented objects at various distances and angles. The most effective minimal distance detection is $20 \mathrm{~cm}$ from above and maximum distance detection is $90 \mathrm{~cm}$ at $45^{\circ}$ and $90^{\circ}$ angles. It is proven that markerless tracking using augmented reality is applicable and more effective for a campus visualization.

\section{$6 \quad$ References}

[1] A. kumar. P and M. R, "Prospects of Augmented Reality in Physical Stores's using Shopping Assistance App,” Procedia Comput. Sci., 2020, https://doi.org/10.1016/j.procs.2020.05.074

[2] A. Poushneh, "Augmented reality in retail: A trade-off between user's control 1,,,,,,,,of access to personal information and augmentation quality," J. Retail. Consum. Serv., 2018, https://doi.org/10.1016/j.jretconser.2017.12.010

[3] S. Yang, J. R. Carlson, and S. Chen, "How augmented reality affects advertising effectiveness: The mediating effects of curiosity and attention toward the ad," J. Retail. Consum. Serv., 2020, https://doi.org/10.1016/j.jretconser.2019.102020

[4] T. Masood and J. Egger, "Adopting augmented reality in the age of industrial digitalisation," Comput. Ind., 2020, doi: 10.1016/j.compind.2019.07.002.

[5] S. Boonbrahm, P. Boonbrahm, and C. Kaewrat, "The use of marker-based augmented reality in space measurement," in Procedia Manufacturing, 2020, https://doi.org/10.1016/j.promfg. 2020.02 .081

[6] A. K. Dash, S. K. Behera, D. P. Dogra, and P. P. Roy, "Designing of marker-based augmented reality learning environment for kids using convolutional neural network architecture,” Displays, 2018, https://doi.org/10.1016/j.displa.2018.10.003

[7] M. M. Seran Bria, G. P. Kusuma, and L. K. Putera Suryapranata, "Promoting Timor Leste's Tais Cloth Using Mobile Augmented Reality Application," in Procedia Computer Science, 2018, https://doi.org/10.1016/j.procs.2018.08.216

[8] D. Rohendi and Y. Wihardi, "Learning Three-Dimensional Shapes in Geometry Using Mobile-Based Augmented Reality," Int. J. Interact. Mob. Technol., vol. 14, no. 09, p. 48, 2020, https://doi.org/10.3991/ijim.v14i09.13035

[9] I.W.A.Indrawan, I.P.A.Bayupati, and D.P.S.Putri, "Markerless Augmented Reality Utilizing Gyroscope to Demonstrate the Position of Dewata Nawa Sanga," Int. J. Interact. Mob. Technol., vol. 12, no. 1, pp. 19-36, 2018. https://doi.org/10.3991/ijim.v12i1.7527

[10] M. S. A. El-Seoud and I. A. T. F. Taj-Eddin, "An android augmented reality application for retail fashion shopping," Int. J. Interact. Mob. Technol., 2019, https://doi.org/10.3991/ ijim.v13i01.9898

[11] J. Polvi, T. Taketomi, G. Yamamoto, A. Dey, C. Sandor, and H. Kato, "SlidAR: A 3D positioning method for SLAM-based handheld augmented reality," Comput. Graph., 2016, https://doi.org/10.1016/j.cag.2015.10.013

[12] R. Egodagamage and M. Tuceryan, "Distributed monocular visual SLAM as a basis for a collaborative augmented reality framework," Comput. Graph., 2018, https://doi.org/10. 1016/j.cag.2018.01.002

[13] P. Haynes, S. Hehl-Lange, and E. Lange, "Mobile Augmented Reality for Flood Visualisation,” Environ. Model. Softw., 2018, https://doi.org/10.1016/j.envsoft.2018.05.012 
[14] D. Ramadasan, M. Chevaldonne, and T. Chateau, "Real-time SLAM for static multi-objects learning and tracking applied to augmented reality applications," in 2015 IEEE Virtual Reality Conference, VR 2015 - Proceedings, 2015, https://doi.org/10. 1109/vr.2015.7223398

[15] J. C. G. Vargas, R. Fabregat, A. Carrillo-Ramos, and T. Jové, "Survey: Using augmented reality to improve learning motivation in cultural heritage studies," Appl. Sci., 2020, https://doi.org/10.3390/app10030897

[16] M. Trunfio and S. Campana, "A visitors' experience model for mixed reality in the museum," Current Issues in Tourism. 2020, https://doi.org/10.1080/13683500. 2019.1586847

\section{$7 \quad$ Authors}

Budi Arifitama is an Assistant Professor at the Department of Informatics, Universitas Trilogi. He has published computer science papers mostly in reputable international journals and conferences related to augmented reality and human-computer interaction. Email: budiarif@trilogi.ac.id

Ghali Hanan \& Muhamad Halim Rofiqi works as an Assistant Lecturer at the Immersive Technology Laboratory, Universitas Trilogi.

Article submitted 2020-12-22. Resubmitted 2021-03-10. Final acceptance 2021-03-18. Final version published as submitted by the authors. 